ORIGINAL

\title{
Comportamento de variáveis cardiorrespiratórias em idosos e jovens durante 15 minutos de recuperação após esforços de diferentes intensidades
}

\author{
Paulo T.V. Farinatti ${ }^{1}$, Jacques H.P. Vanfraechem ${ }^{2}$ e Walace D. Monteiro ${ }^{3}$
}

\section{RESUMO}

Sabe-se pouco sobre os efeitos do envelhecimento na recuperação pós-esforço (RP). O estudo observou a RP em 15 idosos (GI, idade $=61 \pm 1$ anos) e 15 jovens (GJ, idade $=22 \pm 2$ anos) após atividades de três intensidades (IE) em cicloergômetro. Realizaram-se testes máximos, com incremento de $30 \mathrm{~W} / \mathrm{min}$ para GJ e de $25 \mathrm{~W} / \mathrm{min}$ após detecção de steadystate para GI. Posteriormente, os grupos pedalaram a $40 \%$ e $75 \%$ da carga máxima, respectivamente, 25 e 15 minutos. Foram acompanhados $\dot{\mathrm{VO}}_{2}, \dot{\mathrm{V}} \mathrm{VO}_{2}, \mathrm{~V}_{\mathrm{E}}$ e $\mathrm{FC}$ nos primeiros 15 minutos da RP nas três IE. O tratamento dos resultados compreendeu: a) teste de ajustamento das curvas experimentais a equações com uma ou duas exponenciais; b) cálculo do valor dos componentes para a equação mais ajustada; c) análise das constantes extraídas. Os desvios de ajustamento foram inferiores para uma curva de duas exponenciais, definida por integral de tempo na forma $\mathrm{A} / \alpha+\mathrm{B} / \beta$. $\mathrm{A} / \alpha$ designa a componente rápida da recuperação e $\mathrm{B} / \beta$ a lenta. Quando comparados os grupos, GI mostrou constantes maiores que GJ, evidenciando recuperação mais lenta nas duas fases. Subdividindo os componentes, em GI e GJ as constantes de velocidade de recuperação rápida $(1 / \alpha)$ para $\dot{\mathrm{VO}}_{2}$ e $\dot{\mathrm{V}} \mathrm{CO}_{2}$ foram semelhantes nas três IE, enquanto para a constante lenta $(1 / \beta)$, os valores para GI indicaram maior dependência em relação à carga. A recuperação da FC revelou-se extremamente dependente da IE para GJ. Para GI isso foi menos evidente, talvez

1. Instituto de Educação Física e Desportos, Universidade do Estado do Rio de Janeiro, Rio de Janeiro - RJ.

2. Institut Supérieur d'Education Physique et de Kinesitherapie, Université Libre de Bruxelles, Bruxelas - Bélgica.

3. Núcleo do Instituto de Ciências da Atividade Física, Força Aérea Brasileira, Rio de Janeiro - RJ

Endereço para correspondência:

Paulo T.V. Farinatti

Instituto de Educação Física e Desportos/

Coordenadoria de Pós-Graduação

Rua São Francisco Xavier, 524, sala 9.122, bloco F, Maracanã

20550-013 - Rio de Janeiro, RJ

E-mail: farinatti@uol.com.br

Fax: (021) 587-7862 por menor possibilidade de elevação da FC. A V $\mathrm{E}_{\mathrm{E}}$ em GJ comportou-se de forma similar ao $\dot{\mathrm{VO}}_{2}$ e $\dot{\mathrm{V} C O}$. Porém, para GI as constantes de tempo foram mais lentas, mais associadas à IE que os demais parâmetros. Conclui-se: a) pode existir uma constante comum para a chamada 'fase alática' da curva de recuperação do $\dot{\mathrm{VO}_{2}}$ e do $\dot{\mathrm{VCO}_{2}}$, independentemente da IE e da idade; b) as diferenças entre GJ e GI podem dever-se às menores potência aeróbia máxima, termorregulação e eficácia do tampão respiratório nos idosos.

Palavras-chave: Atividade física. Teste de esforço. Idosos. Envelhecimento. Recuperação. Exercício.

Apoio financeiro: CNPq (200063/94-4)

\section{ABSTRACT}

Behavior of cardiorespiratory variables in elderly and young individuals during 15 minute recovery after efforts of varying intensities

Few data are available about post-exercise recovery $(P R)$ and the aging process. Recovery of $\dot{\mathrm{V}} \mathrm{O}_{2}, \dot{\mathrm{V} C \mathrm{O}_{2}}, V_{E}$ and $\mathrm{HR}$ was observed at different exercise loads (EL) on cyclo-ergometer. A group of 10 young (GJ, age $=22 \pm 2$ years) and 10 elderly subjects (GI, age $=61 \pm 1$ years) performed maximal tests. A $30 \mathrm{~W} / \mathrm{min}$ load incremental was applied to GJ and a $25 \mathrm{~W} / \mathrm{min}$ after steady-state for GI. Also, subjects performed exercise at $40 \%$ and $75 \%$ of peak $\dot{V} \mathrm{O}_{2}$, during 25 and $15 \mathrm{~min}$ utes respectively. $\dot{V} O_{2}, \dot{V} C O_{2}, V_{E}$ and $H R$ were measured during the first 15 minutes of PR for the three EL. Results analysis was made by: a) testing experimental data residuals for one and two exponential equations; b) determining the better equation components; c) analysis of extracted constants. Deviations were smaller for a two exponential function, defined by $S={ }_{0} \int^{\infty} x(t) d t=A / \alpha+B / \beta . A / \alpha$ designs $P R$ fast component and $B / \beta$ the slower one. When groups were compared, GI had higher constants than GJ, showing a slower recovery at both phases. GI and GJ constants for PR fast phase (1/ $\alpha)$ were similar at all EI for $\dot{\mathrm{VO}}{ }_{2}$ and $\dot{\mathrm{VCO}} \mathrm{O}_{2}$. Results suggested a greater dependence of exercise load for PR slower constant (1/ $\beta$ ) in GI. HR recovery has shown to be very much associated to EL in GJ, with time course PR being directly affected by exer- 
cise load. The same results were not observed for GI, maybe because of elderly limited HR upward drift potential. Data for $V_{E}$ recovery were similar to those observed for $\dot{\mathrm{V}} \mathrm{O}_{2}$ and $\dot{\mathrm{V}} \mathrm{CO}_{2}$ in GJ. However, GI constants were slower, more associated to $E L$ than the other parameters. The authors conclude that: a) there can be a common constant for the 'alatic phase' of $P R$ for $\dot{V} \mathrm{O}_{2}$ and $\dot{\mathrm{V}} \mathrm{CO}_{2}$, independent of EL and age; $b$ ) differences between GJ and GI can be due to lower maximal aerobic capacity, thermoregulation and age-related reduced $\mathrm{CO}_{2}$ chemosensitivity, delaying elimination of the exercise-induced $\mathrm{CO}_{2}$ load.

Key words: Physical activity. Effort test. Elderly. Aging. Recovery. Exercise.

Fellowship: CNPq (200063/94-4)

\section{INTRODUÇÃO}

Os padrões de comportamento das variáveis cardiorrespiratórias durante o exercício têm sido bastante estudados com referência à idade, ao sexo e ao nível de treinamento ${ }^{1-6}$. No entanto, como comentam Williams e Horvath ${ }^{7}$, a quantidade de estudos que tratam do período de recuperação pós-esforço é sensivelmente menor, apesar das informações potencialmente úteis que poderiam advir de tal observação. Por exemplo, sabese que a prescrição de certos tipos de programas de exercício, assim como o controle do nível de treinamento, baseiam-se freqüentemente na relação entre a intensidade do esforço aplicado e a duração do período de recuperação $0^{8,9}$.

A variável mais estudada durante a recuperação é, sem dúvida, o consumo de oxigênio $\left(\dot{\mathrm{V}}_{2}\right)^{9-14}$. Já nos anos 20 , Hill et $a l .{ }^{15,16}$ descreveram sua evolução e demonstraram que, depois de uma atividade muscular intensa, o retorno às condições de repouso compreendia dois componentes, um rápido e outro mais lento. Posteriormente, Margaria et al. ${ }^{17}$, Henry ${ }^{18}$ e Henry e De Moor ${ }^{19}$ estabeleceriam que o $\mathrm{V}_{2}$ durante a recuperação, uma vez deduzido o consumo de repouso, poderia ser descrito pela soma de duas exponenciais: $\mathrm{X}(\mathrm{t})=\mathrm{Ae}^{-\alpha t}+\mathrm{Be}^{-\beta t}$. Segundo Margaria et al..$^{20}$, a exponencial de declínio rápido representaria uma fase alática da recuperação, enquanto a exponencial lenta descreveria uma fase lática. Katch et al. ${ }^{21} \mathrm{e}$ $\mathrm{Katch}^{22}$ retomaram esses estudos iniciais e procuraram quantificar os parâmetros dessa equação. No que se refere a outras variáveis cujo comportamento é afetado pelo exercício, como o gás carbônico expirado $\left(\mathrm{V} \mathrm{CO}_{2}\right)$, a ventilação pulmonar $\left(\mathrm{V}_{\mathrm{E}}\right)$ e a freqüência cardíaca (FC), pesquisadores como $\mathrm{Joye}^{23} \mathrm{e}$ Chick et al. ${ }^{24}$ demonstraram que tendem a ser descritas por equações do mesmo tipo que a do $\dot{\mathrm{VO}}_{2}$.

A quase totalidade dos dados disponíveis, contudo, diz respeito a indivíduos jovens ou de meia-idade. A carência de informações sobre o comportamento de parâmetros cardiorrespiratórios durante a recuperação pós-esforço é sensivelmente maior quando se trata de sujeitos de mais de 60 anos. Em um dos poucos estudos que se podem localizar, Chick et al. ${ }^{24} \mathrm{ob}-$ servaram o comportamento do $\dot{\mathrm{VO}}_{2}, \dot{\mathrm{V}} \mathrm{CO}_{2}$ e da $\mathrm{V}_{\mathrm{E}}$ em indivíduos jovens e idosos. Os resultados obtidos indicaram diminuição geral da velocidade de recuperação com a idade, mas retorno similar aos valores basais após 10 minutos de repouso. Contudo, faltam dados para que se possam considerar esses resultados como norma geral. Isso posto, o objetivo deste estudo foi observar quatro variáveis $\left(\dot{\mathrm{V}}_{2}, \dot{\mathrm{VCO}}_{2}, \mathrm{~V}_{\mathrm{E}}\right.$ e FC) durante os primeiros 15 minutos de repouso subsequientes a diferentes intensidades de esforço (IE), em indivíduos idosos e jovens ativos.

\section{MATERIAL E MÉTODOS}

Trinta homens, sendo 15 jovens (grupo jovem - GJ) (idade $=22 \pm 2$ anos) e 15 idosos (grupo idoso - GI) (idade $=61 \pm 1$ anos) participaram voluntariamente do estudo. Eram do curso de Educação Física da Universidade Livre de Bruxelas (GJ) ou participantes de programa de atividades físicas daquela Universidade (GI). Todos os participantes assinaram um termo de consentimento informado, de acordo com as recomendações da Convenção de Helsinque.

Os voluntários foram submetidos a testes de esforço máximo em cicloergômetro de frenagem eletromagnética. O protocolo de avaliação incluiu: a) três minutos de aquecimento a 5 watts; b) uma fase de trabalho, começando a 25 watts para o GI e a 30 watts para o GJ. O incremento de carga para GJ foi feito a cada minuto, enquanto para GI se optou pela detecção prévia de um estado de equilíbrio (steady-state). Ainda que o cicloergômetro mantivesse constantes as cargas para uma frequiência de pedalagem entre 30 e $300 \mathrm{rpm}$, um ritmo de $60 \mathrm{rpm}$ era sugerido para o conforto do avaliado. $\mathrm{O}$ teste era interrompido na presença de sinais de intolerância ao esforço, de acordo com as recomendações do $\mathrm{ACSM}^{25}$.

O teste era considerado máximo se pudesse satisfazer dois de quatro critérios preestabelecidos: a) quociente respiratório $\geq 1,06$; b) freqüência cardíaca de ao menos $80 \%$ do valor máximo previsto para a idade (220 - idade); c) estabilização do $\dot{\mathrm{VO}}_{2}$ apesar de incremento da carga de trabalho (incremento do $\mathrm{VO}_{2} \leq 2,0 \mathrm{ml} \mathrm{x} \mathrm{kg}^{-1} \mathrm{x} \mathrm{min}^{-1}$ entre as duas últimas cargas); d) exaustão voluntária máxima.

A seguir, realizaram-se duas provas submáximas com cargas ajustadas, respectivamente, a $40 \%$ e a $75 \%$ da potência máxima obtida, com duração, respectivamente, de 25 e 15 minutos. Esses percentuais foram escolhidos em razão de provavelmente se situarem em patamares abaixo e acima do limiar anaeróbio dos indivíduos observados ${ }^{11}$. Os testes máximo e submáximo foram realizados com um intervalo de 48 horas. Um período de 15 minutos de recuperação passiva monitorizada seguiu-se a cada um dos testes realizados, com os indivíduos sentados em repouso.

Durante os testes, a pressão arterial foi medida a cada 2 minutos, pelo método auscultatório. O traçado do ECG e a FC 
foram monitorados por um eletrocardiógrafo Rigel modelo 302. Uma derivação bipolar com dois eletrodos colocados na posição $\mathrm{CM}_{5}$ foi utilizada, em função de sua sensibilidade às alterações do segmento ST. O $\mathrm{VO}_{2}$, a $\dot{\mathrm{VCO}}_{2}$ e a $\mathrm{V}_{\mathrm{E}}$ foram medidos durante $15 \mathrm{~min}$ de recuperação, em circuito aberto, por meio da análise direta das frações expiradas de $\mathrm{CO}_{2}$ e $\mathrm{O}_{2}$. A análise dos gases foi feita por analisadores Morgan e o volume ventilatório foi medido por um pneumotacógrafo. As medidas eram corrigidas para condições standard e utilizadas para determinar os valores médios de cada uma das variáveis a cada 30 segundos.

Em resumo, definiram-se três intensidades de esforço distintas para a realização das observações (IE 100\%, IE 75\% e IE $40 \%$ ), em dois grupos amostrais (GJ e GI). O tratamento dos resultados compreendeu: a) teste de ajustamento das curvas experimentais a equações com uma ou duas exponenciais; b) cálculo do valor dos componentes para as equações anteriormente definidas; c) análise dos componentes das constan- tes de velocidade de recuperação extraídas das equações, com referência às intensidades de esforço aplicadas.

\section{RESULTADOS E DISCUSSÃO}

A tabela 1 apresenta os valores médios das variáveis observadas, obtidos no repouso e no esforço máximo, para os dois grupos. Como esperado, os resultados indicam superioridade nos valores obtidos para o grupo jovem durante o esforço, mas similaridade para as condições de repouso. Esses dados são coerentes com o que é geralmente proposto por outros estudos $^{4,6,26}$. Nas tabelas 2 e 3 exibimos os valores médios obtidos para GJ e GI durante a recuperação em vários pontos de corte, uma vez subtraídos os valores de repouso. A análise dos resultados indica que as diferenças de recuperação entre os indivíduos jovens e idosos são maiores à medida que aumentamos a intensidade de esforço relativo, o que nos parece coerente com os dados atualmente disponíveis.

TABELA 1

Valores médios obtidos para os grupos jovem e idoso em repouso e no esforço máximo $(\mathbf{n}=20$ )

\begin{tabular}{|c|c|c|c|c|}
\hline Variável & \multicolumn{2}{|c|}{ GJ $(\bar{X} \pm d p)(n=10)$} & \multicolumn{2}{|c|}{ GI $(\bar{X} \pm d p)(n=10)$} \\
\hline Altura $(\mathrm{cm})$ & \multicolumn{2}{|c|}{$\begin{array}{l}1,75 \pm 0,44 \\
75,6 \pm 5,8\end{array}$} & \multicolumn{2}{|c|}{$\begin{array}{l}1,71 \pm 0,03 \\
71,2 \pm 7,4\end{array}$} \\
\hline & Repouso & Esforço máx (100\%) & Repouso & Esforço máx (100\%) \\
\hline $\begin{array}{l}\dot{\mathrm{VO}}_{2}\left(\mathrm{ml} \times \mathrm{kg}^{-1} \times \mathrm{min}^{-1}\right) \\
\operatorname{VCO}_{2}\left(\mathrm{ml} \mathrm{kg}^{-1} \times \mathrm{min}^{-1}\right) \\
\mathrm{V}_{\mathrm{E}}\left(\mathrm{ml}^{-1} \mathrm{~kg}^{-1} \times \mathrm{min}^{-1}\right) \\
\left.\text { FC (bat } \times \mathrm{min}^{-1}\right) \\
\text { Potência desenvolvida (W) }\end{array}$ & $\begin{aligned} & 4,0 \pm 0,7 \\
& 4,7 \pm 0,5 \\
& 152 \pm 24 \\
& 76 \pm 7 \\
& 0\end{aligned}$ & $\begin{array}{c}52,6 \pm 6,4 \\
61,7 \pm 8,4 \\
1.705 \pm 370 \\
184 \pm 8 \\
236 \pm 28\end{array}$ & $\begin{aligned} & 3,8 \pm 0,3 \\
& 4,5 \pm 0,5 \\
& 138 \pm 16 \\
& 82 \pm 6 \\
& 0\end{aligned}$ & $\begin{array}{c}33,6 \pm 5,1 \\
40,2 \pm 4,3 \\
1.220 \pm 180 \\
156 \pm 6 \\
138 \pm 14\end{array}$ \\
\hline
\end{tabular}

TABELA 2

Valores médios de parâmetros cardiorrespiratórios durante 15 minutos de recuperação (deduzidos os valores de repouso) (grupo jovem, GJ , n = 10)

\begin{tabular}{|c|c|c|c|c|c|c|c|c|c|c|c|c|}
\hline \multirow[t]{2}{*}{$t$ (min) } & \multicolumn{4}{|c|}{ IT = 100\% } & \multicolumn{4}{|c|}{ IT $=75 \%$} & \multicolumn{4}{|c|}{$\mathrm{IT}=\mathbf{4 0} \%$} \\
\hline & $\dot{\mathrm{VO}}_{2}$ & $\dot{\mathrm{v}} \mathrm{CO}_{2}$ & $\mathbf{V}_{E}$ & FC & $\dot{\mathrm{VO}}_{2}$ & $\dot{\mathrm{V}} \mathrm{CO}_{2}$ & $\mathbf{V}_{\mathrm{E}}$ & FC & $\dot{\mathrm{VO}}_{2}$ & $\dot{\mathrm{v}} \mathrm{CO}_{2}$ & $\mathbf{V}_{\mathrm{E}}$ & FC \\
\hline 0 & 48,6 & 57,0 & $1.553,0$ & 108,0 & 32,0 & 33,5 & 887,0 & 86,6 & 17,1 & 17,3 & 402,0 & 38,8 \\
\hline 1 & 23,6 & 38,9 & 968,0 & 86,0 & 16,7 & 19,1 & 486,0 & 58,2 & 7,2 & 6,9 & 180,2 & 14,6 \\
\hline 2 & 7,9 & 16,8 & 495,0 & 59,2 & 5,8 & 9,2 & 251,0 & 40,1 & 2,8 & 3,2 & 72,1 & 7,8 \\
\hline 3 & 5,4 & 11,5 & 360,0 & 44,2 & 4,1 & 6,0 & 184,0 & 32,5 & 0,9 & 1,5 & 34,2 & 5,4 \\
\hline 4 & 4,6 & 9,8 & 310,0 & 38,3 & 2,5 & 3,9 & 132,0 & 28,4 & 1,2 & 1,4 & 37,2 & 3,2 \\
\hline 5 & 3,7 & 7,9 & 249,0 & 32,7 & 1,2 & 3,2 & 118,0 & 27,9 & 0,5 & 1,2 & 27,9 & 2,4 \\
\hline 7 & 2,9 & 5,8 & 197,0 & 28,7 & 1,4 & 1,4 & 51,0 & 25,1 & 0,5 & 0,9 & 15,8 & 3,2 \\
\hline 9 & 2,8 & 4,6 & 172,0 & 26,0 & 1,7 & 1,3 & 60,0 & 21,3 & 0,3 & 0,1 & 13,4 & $-0,4$ \\
\hline 11 & 2,7 & 4,1 & 163,0 & 24,1 & 0,9 & 1,5 & 64,0 & 20,0 & 1,0 & 0,8 & 28,9 & $-2,1$ \\
\hline 15 & 1,8 & 2,2 & 91,0 & 22,3 & 0,8 & 0,6 & 31,0 & 18,2 & 0,1 & 0,4 & 14,2 & $-1,1$ \\
\hline
\end{tabular}

$\dot{\mathrm{VO}}_{2}: \mathrm{ml} \times \mathrm{kg}^{-1} \times \mathrm{min}^{-1} ; \mathrm{VCO}_{2}: \mathrm{ml} \times \mathrm{kg}^{-1} \times \mathrm{min}^{-1} ; \mathrm{V}_{\mathrm{E}}: \mathrm{ml} \times \mathrm{kg}^{-1} \times \mathrm{min}^{-1}$; FC: bat $\times \mathrm{min}^{-1}$ 


\begin{tabular}{|c|c|c|c|c|c|c|c|c|c|c|c|c|}
\hline \multirow{3}{*}{ t (min) } & \multicolumn{11}{|c|}{$\begin{array}{l}\text { TABELA } 3 \\
\text { Valores médios de parâmetros cardiorrespiratórios durante } 15 \text { minutos de } \\
\text { recuperação (deduzidos os valores de repouso) (grupo idoso, } \mathrm{Gl}, \mathbf{n}=10 \text { ) }\end{array}$} & \\
\hline & \multicolumn{4}{|c|}{ IT = 100\% } & \multicolumn{4}{|c|}{ IT $=75 \%$} & \multicolumn{4}{|c|}{$\mathrm{IT}=40 \%$} \\
\hline & $\mathrm{VO}_{2}$ & $\dot{\mathrm{v}} \mathrm{CO}_{2}$ & $\mathbf{V}_{\mathrm{E}}$ & FC & $\mathrm{VO}_{2}$ & $\dot{\mathrm{v}} \mathrm{CO}_{2}$ & $\mathbf{V}_{E}$ & FC & $\mathrm{VO}_{2}$ & $\dot{\mathrm{v}} \mathrm{CO}_{2}$ & $\mathbf{V}_{\mathrm{E}}$ & FC \\
\hline 0 & 29,8 & 35,7 & $1.082,0$ & 74,6 & 24,2 & 25,0 & 834,0 & 58,5 & 14,2 & 14,0 & 501,0 & 49,0 \\
\hline 1 & 21,3 & 20,5 & 796,0 & 64,4 & 13,0 & 14,2 & 421,0 & 52,0 & 8,1 & 7,8 & 248,0 & 38,1 \\
\hline 2 & 8,4 & 12,4 & 392,0 & 55,1 & 6,9 & 7,2 & 238,0 & 39,8 & 4,1 & 3,7 & 101,0 & 16,2 \\
\hline 3 & 6,6 & 9,8 & 321,0 & 46,0 & 3,8 & 4,4 & 170,0 & 33,1 & 1,0 & 1,8 & 70,0 & 12,0 \\
\hline 4 & 5,9 & 7,1 & 287,0 & 38,3 & 2,7 & 3,8 & 132,0 & 28,0 & 1,1 & 1,6 & 51,0 & 8,3 \\
\hline 5 & 5,1 & 5,2 & 234,0 & 35,0 & 2,3 & 3,2 & 111,0 & 25,3 & 0,6 & 1,2 & 32,0 & 6,2 \\
\hline 7 & 3,4 & 3,8 & 200,0 & 31,7 & 2,0 & 2,5 & 80,0 & 22,2 & 0,4 & 0,8 & 26,0 & 4,5 \\
\hline 9 & 2,7 & 2,9 & 172,0 & 27,2 & 1,2 & 1,3 & 72,0 & 18,8 & 0,0 & 0,0 & 12,0 & 2,3 \\
\hline 11 & 2,8 & 2,4 & 155,0 & 24,1 & 1,2 & 1,2 & 51,0 & 14,7 & 1,2 & 1,0 & 12,0 & 1,9 \\
\hline 15 & 2,8 & 2,3 & 88,0 & 22,0 & 1,5 & 1,2 & 32,0 & 14,0 & 0,4 & 0,3 & 7,0 & 0,9 \\
\hline
\end{tabular}

$\mathrm{VO}_{2}: \mathrm{ml} \times \mathrm{kg}^{-1} \times \mathrm{min}^{-1} ; \mathrm{V} \mathrm{CO}_{2}: \mathrm{ml} \times \mathrm{kg}^{-1} \times \mathrm{min}^{-1} ; \mathrm{V}_{\mathrm{E}}: \mathrm{ml} \times \mathrm{kg}^{-1} \times \mathrm{min}^{-1} ; \mathrm{FC}:$ bat $\times \mathrm{min}^{-1}$

TABELA 4

Desvios médios das curvas de recuperação em relação a equações com uma ou duas exponenciais $(n=20)$

\begin{tabular}{|c|c|c|c|c|c|}
\hline \multirow[t]{2}{*}{ Variável } & \multirow[t]{2}{*}{ IE } & \multicolumn{2}{|c|}{$x(t)=A e^{-\alpha t}$} & \multicolumn{2}{|c|}{$x(t)=A e^{-\alpha t}+B e^{-\beta t}$} \\
\hline & & $\begin{array}{c}\text { GJ } \\
(n=10)\end{array}$ & $\begin{array}{c}\text { GI } \\
(n=10)\end{array}$ & $\begin{array}{c}\text { GJ } \\
(n=10)\end{array}$ & $\begin{array}{c}\text { GI } \\
(n=10)\end{array}$ \\
\hline \multirow{3}{*}{$\dot{\mathrm{VO}_{2}}$} & $100 \%$ & $D=2,21$ & $D=2,34$ & $D=0,78$ & $D=0,61$ \\
\hline & $75 \%$ & $D=1,23$ & $D=1,38$ & $D=0,60$ & $D=0,58$ \\
\hline & $40 \%$ & $D=0,53$ & $D=0,55$ & $D=0,47$ & $D=0,43$ \\
\hline \multirow{3}{*}{$\dot{\mathrm{V}} \mathrm{CO}_{2}$} & $100 \%$ & $D=2,78$ & $D=2,89$ & $D=0,77$ & $D=0,81$ \\
\hline & $75 \%$ & $D=1,08$ & $D=1,31$ & $D=0,44$ & $D=0,77$ \\
\hline & $40 \%$ & $D=0,72$ & $D=0,66$ & $D=0,44$ & $D=0,33$ \\
\hline \multirow{3}{*}{$V_{E}$} & $100 \%$ & $D=99,12$ & $D=93,20$ & $D=17,61$ & $D=18,13$ \\
\hline & $75 \%$ & $D=44,56$ & $D=42,56$ & $D=13,71$ & $D=21,30$ \\
\hline & $40 \%$ & $D=22,62$ & $D=24,71$ & $D=14,28$ & $D=12,42$ \\
\hline \multirow{3}{*}{ FC } & $100 \%$ & $D=10,10$ & $D=8,20$ & $D=2,00$ & $D=2,80$ \\
\hline & $75 \%$ & $D=8,40$ & $D=8,40$ & $D=1,40$ & $D=1,90$ \\
\hline & $40 \%$ & $D=2,30$ & $D=3,50$ & $D=1,80$ & $D=1,80$ \\
\hline
\end{tabular}

Determinou-se a seguir o ajustamento da curva experimental com uma ou duas exponenciais, para cada uma das variáveis observadas nas três IE. Os desvios foram determinados com base em equação de ajustamento utilizada por Joye ${ }^{23}$ : D $=\left[\sum_{\mathrm{i}} \mathrm{d}_{\mathrm{i}}^{2} /\left(\mathrm{N}_{\mathrm{p}}-\mathrm{N}_{\mathrm{a}}\right)\right]^{1 / 2}$, onde $\mathrm{d}_{\mathrm{i}}=$ desvio referente ao $1^{\mathrm{o}}$ ponto, $\mathrm{N}_{\mathrm{p}}$ = número de pontos e $\mathrm{N}_{\mathrm{a}}$ = número de constantes ajustadas . Os resultados discriminados de acordo com a variável e intensidade encontram-se na tabela 4. Como esperado, em todos os casos os desvios relativos aos dados brutos revelaram-se menores quando o ajustamento foi realizado por meio de uma curva representando a soma de duas exponenciais. Dessa for- ma, a análise dos dados foi feita com base em uma equação do tipo $\mathrm{x}(\mathrm{t})=\mathrm{Ae}^{-\alpha \mathrm{t}}+\mathrm{Be}^{-\beta \mathrm{t}}$, onde $\mathrm{A}=$ valor do componente rápido no tempo zero; $\mathrm{B}=$ valor do componente lento no tempo zero; $\alpha=$ constante de velocidade da primeira exponencial; $\beta=$ constante de velocidade da segunda exponencial; $\mathrm{e}=$ base de logaritmos neperianos; $\mathrm{t}=$ tempo. Calcularam-se então os componentes e constantes para cada uma das variáveis, em ambos os grupos de idade. Os resultados obtidos são apresentados nas tabelas 5 e 6.

A recuperação total de cada uma das variáveis é dada pela área delimitada pelas curvas. Assim, calculou-se a integral correspondente às duas exponenciais obtidas, ou seja: $\mathrm{S}=\int_{0}^{\infty}$ $\mathrm{x}(\mathrm{t}) \mathrm{dt}=\mathrm{A} / \alpha+\mathrm{B} / \beta$. As partes respectivas descritas por cada um dos fatores dessa equação encontram-se na tabela 7.

Nota-se que, se os valores de B são inferiores aos valores de A (tabelas 5 e 6), quando observamos a área relativa à recuperação total o fator relativo ao componente lento do processo de recuperação $(B / \beta)$ é, na maioria absoluta dos casos, mais pronunciado que o fator associado ao componente rápido $(\mathrm{A} / \alpha)$. Percebe-se ainda que, quanto maior a intensidade do esforço, maior o percentual de contribuição da fase lenta de recuperação para a normalização das variáveis observadas, fato que independe da idade dos sujeitos ${ }^{8,23,24}$. Em termos percentuais, o envelhecimento parece não acarretar alterações significativas para a participação relativa de cada uma das fases da recuperação total dessas variáveis, ao menos no que diz respeito a nossa amostra.

\section{Recuperação do consumo de oxigênio}

Os valores da constante de velocidade $\alpha$ são similares nas três intensidades de esforço analisadas, para ambos os grupos etários (tabelas 5 e 6). O valor da relação $1 / \alpha$ foi, em todas as situações, próximo de $1 \mathrm{~min}$. Considerando os desvios-padrões 
TABELA 5

Ajustamento das curvas de recuperação segundo a equação $x(t)=A e^{-\alpha t}+B e^{-\beta t: ~ v a l o r e s ~}$ dos componentes A, B, $\alpha$ e $\beta$ nas três intensidades de esforço (grupo jovem, $G$ J , $n=10$ )

\begin{tabular}{|c|c|c|c|c|}
\hline IE & $\dot{\mathrm{V}} \mathrm{O}_{2}\left(\mathrm{ml} \times \mathrm{kg}^{-1} \times \mathrm{min}^{-1}\right)$ & $\dot{\mathrm{V}} \mathrm{CO}_{2}\left(\mathrm{ml} \times \mathrm{kg}^{-1} \times \mathrm{min}^{-1}\right)$ & $V_{E}\left(m l \times k^{-1} \times \min ^{-1}\right)$ & FC (bat $x$ min-1) $^{-1}$ \\
\hline $100 \%$ & $\begin{array}{l}A=43,82 \pm 0,79 \\
B=3,48 \pm 0,51\end{array}$ & $\begin{array}{l}A=49,51 \pm 1,16 \\
B=12,06 \pm 1,22\end{array}$ & $\begin{array}{l}A=1.238,90 \pm 21,57 \\
B=342,58 \pm 19,35\end{array}$ & $\begin{array}{l}A=78,00 \pm 2,00 \\
B=28,00 \pm 2,00\end{array}$ \\
\hline $75 \%$ & $\begin{array}{l}A=30,29 \pm 0,66 \\
B=2,39 \pm 0,55\end{array}$ & $\begin{array}{l}A=26,33 \pm 0,99 \\
B=6,88 \pm 1,01\end{array}$ & $\begin{array}{l}A=707,32 \pm 18,84 \\
B=178,94 \pm 17,43\end{array}$ & $\begin{array}{l}A=58,00 \pm 1,00 \\
B=31,00 \pm 2,00\end{array}$ \\
\hline $40 \%$ & $\begin{array}{l}A=15,58 \pm 0,48 \\
B=0,59 \pm 0,27\end{array}$ & $\begin{array}{l}A=15,17 \pm 0,49 \\
B=1,83 \pm 0,46\end{array}$ & $\begin{array}{l}A=384,21 \pm 13,14 \\
B=26,28 \pm 7,16\end{array}$ & $\begin{array}{l}A=31,00 \pm 3,00 \\
B=10,00 \pm 2,00\end{array}$ \\
\hline & \multicolumn{4}{|c|}{$\alpha\left(\min ^{-1}\right)[1 / \alpha]$} \\
\hline & $\dot{\mathrm{VO}}_{2}$ & $\dot{\mathrm{V}} \mathrm{CO}_{2}$ & $\mathbf{V}_{\mathbf{E}}$ & FC \\
\hline $100 \%$ & $1,098 \pm 0,045[0,91]$ & $0,887 \pm 0,041[1,13]$ & $0,828 \pm 0,028[1,21]$ & $0,489 \pm 0,030[2,01]$ \\
\hline $75 \%$ & $0,997 \pm 0,048[1,00]$ & $0,948 \pm 0,045[1,05]$ & $1,028 \pm 0,049[0,97]$ & $0,866 \pm 0,048[1,15]$ \\
\hline \multirow[t]{2}{*}{$40 \%$} & $1,034 \pm 0,068[0,97]$ & $1,128 \pm 0,087[0,89]$ & $0,999 \pm 0,068[1,00]$ & $1,492 \pm 0,287[0,67]$ \\
\hline & \multicolumn{4}{|c|}{$\beta\left(\min ^{-1}\right)[1 / \beta]$} \\
\hline $100 \%$ & $0,039 \pm 0,010[25,64]$ & $0,118 \pm 0,010 \quad[8,48]$ & $0,082 \pm 0,004[12,20]$ & $0,014 \pm 0,004[71,43]$ \\
\hline $75 \%$ & $0,059 \pm 0,019[16,99]$ & $0,170 \pm 0,021 \quad[5,88]$ & $0,120 \pm 0,011 \quad[8,33]$ & $0,037 \pm 0,004[27,03]$ \\
\hline $40 \%$ & $0,055 \pm 0,044[18,18]$ & $0,096 \pm 0,028[10,42]$ & $0,020 \pm 0,022[50,00]$ & $0,211 \pm 0,058 \quad[4,74]$ \\
\hline
\end{tabular}

\section{TABELA 6}

Ajustamento das curvas de recuperação segundo a equação $x(t)=A e^{-\alpha t}+B e^{-\beta t}$ : valores dos componentes A, B, $\alpha$ e $\beta$ nas três intensidades de esforço (grupo idoso, $\mathbf{G l}, \mathbf{n}=10$ )

\begin{tabular}{|c|c|c|c|c|}
\hline IE & $\dot{\mathrm{V}} \mathrm{O}_{2}\left(\mathrm{ml} \times \mathrm{kg}^{-1} \times \mathrm{min}^{-1}\right)$ & $\dot{\mathrm{V}} \mathrm{CO}_{2}\left(\mathrm{ml} \mathrm{x} \mathrm{kg}{ }^{-1} \times \mathrm{min}^{-1}\right)$ & $V_{E}\left(m \mathrm{l} \times \mathrm{kg}^{-1} \times \mathrm{min}^{-1}\right)$ & FC (bat x min-1) \\
\hline $100 \%$ & $\begin{array}{l}A=48,68 \pm 0,83 \\
B=3,01 \pm 0,49\end{array}$ & $\begin{array}{l}A=57,80 \pm 0,74 \\
B=9,95 \pm 0,44\end{array}$ & $\begin{array}{l}A=817,35 \pm 20,52 \\
B=242,00 \pm 10,42\end{array}$ & $\begin{array}{l}A=61,45 \pm 2,00 \\
B=23,95 \pm 1,00\end{array}$ \\
\hline $75 \%$ & $\begin{array}{l}A=44,79 \pm 0,57 \\
B=2,11 \pm 0,35\end{array}$ & $\begin{array}{l}A=30,10 \pm 0,63 \\
B=5,70 \pm 0,28\end{array}$ & $\begin{array}{l}A=443,13 \pm 20,31 \\
B=120,40 \pm 17,24\end{array}$ & $\begin{array}{l}A=29,70 \pm 1,00 \\
B=13,41 \pm 1,00\end{array}$ \\
\hline $40 \%$ & $\begin{array}{l}A=18,52 \pm 0,39 \\
B=0,84 \pm 0,21\end{array}$ & $\begin{array}{l}A=17,30 \pm 0,29 \\
B=3,58 \pm 0,22\end{array}$ & $\begin{array}{l}A=310,94 \pm 12,29 \\
B=179,48 \pm 10,98\end{array}$ & $\begin{array}{l}A=13,12 \pm 1,00 \\
B=1,14 \pm 0,01\end{array}$ \\
\hline
\end{tabular}

\begin{tabular}{|c|c|c|c|c|}
\hline & \multicolumn{4}{|c|}{$\alpha\left(\min ^{-1}\right)[1 / \alpha]$} \\
\hline & $\dot{\mathrm{VO}}_{2}$ & $\dot{\mathrm{VCO}}_{2}$ & $\mathbf{V}_{\mathrm{E}}$ & FC \\
\hline $100 \%$ & $1,031 \pm 0,040[0,97]$ & $0,978 \pm 0,038[1,02]$ & $0,537 \pm 0,021[1,86]$ & $0,361 \pm 0,018[2,77]$ \\
\hline $75 \%$ & $1,089 \pm 0,039[0,92]$ & $1,003 \pm 0,060[0,99]$ & $0,633 \pm 0,030[1,58]$ & $0,410 \pm 0,032[2,44]$ \\
\hline \multirow[t]{2}{*}{$40 \%$} & $1,011 \pm 0,061[0,99]$ & $1,147 \pm 0,091[0,87]$ & $0,780 \pm 0,044[1,28]$ & $0,570 \pm 0,013[1,75]$ \\
\hline & \multicolumn{4}{|c|}{$\beta\left(\min ^{-1}\right)[1 / \beta]$} \\
\hline $100 \%$ & $0,032 \pm 0,010[31,25]$ & $0,099 \pm 0,013[10,10]$ & $0,055 \pm 0,020[18,18]$ & $0,013 \pm 0,005[76,92]$ \\
\hline $75 \%$ & $0,050 \pm 0,015[20,00]$ & $0,128 \pm 0,026 \quad[7,81]$ & $0,079 \pm 0,018[12,66]$ & $0,015 \pm 0,002[66,67]$ \\
\hline $40 \%$ & $0,062 \pm 0,053[16,13]$ & $0,124 \pm 0,038 \quad[8,06]$ & $0,128 \pm 0,042 \quad[7,81]$ & $0,022 \pm 0,053[45,46]$ \\
\hline
\end{tabular}


encontrados, é razoável pensar que exista uma constante comum para a chamada 'fase alática' da curva de recuperação, independentemente da intensidade do esforço realizado e mesmo da faixa etária. No esforço máximo, $1 / \alpha=54,6$ s (grupo jovem) e 58,2s (grupo idoso). A uma IE de $75 \%$ do máximo, $1 / \alpha=60$ s (grupo jovem) e 55,2s (grupo idoso). Finalmente, a $40 \%$ da IE máxima, $1 / \alpha=58,2 \mathrm{~s}$ (grupo jovem) e 59,4s (grupo idoso).

Os resultados obtidos para as constantes de velocidade $\beta$, associados ao componente lento da recuperação, parecem ser mais influenciados pela intensidade do esforço. Em 100\% IE, a relação $1 / \beta$ foi de $25,6 \mathrm{~min}$ para os jovens e de $31,3 \mathrm{~min}$ para os idosos. Os valores para as intensidades submáximas foram de $17,0 \mathrm{~min}$ (75\%, grupo jovem), 20,0 min (75\%, grupo idoso), $18,2 \mathrm{~min}$ (40\%, grupo jovem) e $16,1 \mathrm{~min}$ (40\%, grupo idoso). Além disso, a influência da intensidade do esforço na fase lenta de recuperação parece ter sido mais sentida pelo grupo idoso. Enquanto neste último grupo as diferenças puderam ser identificadas para as três IE testadas, no grupo jovem a constante de velocidade foi marcadamente mais lenta para o esforço a $100 \%$, enquanto para as IE de $75 \%$ e $40 \%$ o tempo de recuperação foi similar. Os resultados exibidos na tabela 7 apontam na mesma direção. Nota-se que, contrariamente ao observado para o componente rápido, em que os valores de A/ $\alpha$ foram similares a IE $100 \%$ e IE $75 \%$, o componente lento $(B / \beta)$ teve valores bem distintos para cada uma das IE.

Finalmente, cumpre mencionar que $\beta$ e $\mathrm{B} / \beta$ tenderam a ser sistematicamente maiores para o grupo idoso. Isso é uma evidência de que a segunda fase de recuperação tem sua duração aumentada à medida que envelhecemos. $\mathrm{O}$ fato de a potência aeróbia diminuir com a idade $\mathrm{j}^{5,6,26,27}$, com declínio concomitante da capacidade de metabolização do lactato, de regulação térmica e wash-out de catecolaminas circulantes, pode ser uma explicação para esse comportamento. Dawson et al. ${ }^{11}$,

TABELA 7

Recuperação total dos componentes rápidos e lentos dados pela integral $S={ }_{0}{ }^{\infty} x(t) d t=A \alpha+B / \beta$ para $\dot{\mathrm{VO}}_{2}, \mathrm{VCO}_{2}, \mathrm{~V}_{\mathrm{E}}$ e FC em 15min de recuperação após esforço de diferentes intensidades $(n=20)$

\begin{tabular}{|c|c|c|c|c|}
\hline GJ $(n=10)$ & IE & $\begin{array}{l}\text { Componente } \\
\text { rápido } A / \alpha\end{array}$ & $\begin{array}{c}\text { Componente } \\
\text { lento } B / \beta\end{array}$ & $\begin{array}{c}\text { Recuperação } \\
\text { total }\end{array}$ \\
\hline$\dot{\mathrm{VO}}_{2}\left(\mathrm{ml} \times \mathrm{kg}^{-1} \times \mathrm{min}^{-1}\right)$ & $\begin{array}{r}100 \% \\
75 \% \\
40 \%\end{array}$ & $\begin{array}{l}39,91(30,90 \%) \\
30,38(42,86 \%) \\
15,07(58,41 \%)\end{array}$ & $\begin{array}{l}89,23(69,10 \%) \\
40,51(57,14 \%) \\
10,73(41,59 \%)\end{array}$ & $\begin{array}{r}129,14 \\
70,89 \\
25,08\end{array}$ \\
\hline $\mathrm{VCO}_{2}\left(\mathrm{ml} \times \mathrm{kg}^{-1} \times \mathrm{min}^{-1}\right)$ & $\begin{array}{r}100 \% \\
75 \% \\
40 \%\end{array}$ & $\begin{array}{l}55,82(35,32 \%) \\
27,77(40,70 \%) \\
13,45(41,37 \%)\end{array}$ & $\begin{array}{r}102,20(64,68 \%) \\
40,47(59,30 \%) \\
19,06(58,63 \%)\end{array}$ & $\begin{array}{r}158,02 \\
68,24 \\
32,51\end{array}$ \\
\hline$V_{E}\left(m l \times k g^{-1} \times \min ^{-1}\right)$ & $\begin{array}{r}100 \% \\
75 \% \\
40 \%\end{array}$ & $\begin{array}{r}1.496,26(26,37 \%) \\
688,05(31,57 \%) \\
384,58(22,64 \%)\end{array}$ & $\begin{array}{l}4.177,80(73,63 \%) \\
1.491,17(68,43 \%) \\
1.314,00(77,36 \%)\end{array}$ & $\begin{array}{l}5.674,06 \\
2.179,22 \\
1.698,58\end{array}$ \\
\hline FC (bat $x$ min $\left.^{-1}\right)$ & $\begin{array}{r}100 \% \\
75 \% \\
40 \%\end{array}$ & $\begin{array}{rr}159,51 & (7,39 \%) \\
66,97 & (7,40 \%) \\
20,78 & (30,48 \%)\end{array}$ & $\begin{array}{r}2.000,00(92,61 \%) \\
837,84(92,60 \%) \\
47,39(69,52 \%)\end{array}$ & $\begin{array}{r}2.159,51 \\
904,81 \\
68,17\end{array}$ \\
\hline GI $(n=10)$ & IE & $\begin{array}{l}\text { Componente } \\
\text { rápido } A / \alpha\end{array}$ & $\begin{array}{c}\text { Componente } \\
\text { lento } B / \beta\end{array}$ & $\begin{array}{c}\text { Recuperação } \\
\text { total }\end{array}$ \\
\hline$\dot{\mathrm{V}}_{2}\left(\mathrm{ml} \times \mathrm{kg}^{-1} \times \mathrm{min}^{-1}\right)$ & $\begin{array}{r}100 \% \\
75 \% \\
40 \%\end{array}$ & $\begin{array}{l}47,22(33,40 \%) \\
41,13(49,39 \%) \\
18,32(57,56 \%)\end{array}$ & $\begin{array}{l}94,16(66,60 \%) \\
42,14(50,61 \%) \\
13,51(42,44 \%)\end{array}$ & $\begin{array}{r}141,38 \\
83,27 \\
31,83\end{array}$ \\
\hline $\mathrm{VCO}_{2}\left(\mathrm{ml} \times \mathrm{kg}^{-1} \times \mathrm{min}^{-1}\right)$ & $\begin{array}{r}100 \% \\
75 \% \\
40 \%\end{array}$ & $\begin{array}{l}59,10(37,10 \%) \\
30,01(40,24 \%) \\
15,08(34,30 \%)\end{array}$ & $\begin{array}{r}100,21(62,90 \%) \\
44,56(59,76 \%) \\
28,89(65,70 \%)\end{array}$ & $\begin{array}{r}159,31 \\
74,57 \\
43,97\end{array}$ \\
\hline$V_{E}\left(\mathrm{ml} \times \mathrm{kg}^{-1} \times \mathrm{min}^{-1}\right)$ & $\begin{array}{r}100 \% \\
75 \% \\
40 \%\end{array}$ & $\begin{array}{r}1.522,06(25,70 \%) \\
700,04(31,48 \%) \\
398,64(22,14 \%)\end{array}$ & $\begin{array}{l}4.400,02(74,30 \%) \\
1.524,05(68,52 \%) \\
1.402,20(77,86 \%)\end{array}$ & $\begin{array}{l}5.922,08 \\
2.224,09 \\
1.800,84\end{array}$ \\
\hline FC (bat $\left.x \min ^{-1}\right)$ & $\begin{array}{r}100 \% \\
75 \% \\
40 \%\end{array}$ & $\begin{array}{rr}170,22 & (8,46 \%) \\
72,45 & (7,49 \%) \\
23,01 & (30,67 \%)\end{array}$ & $\begin{array}{r}1.842,44(91,54 \%) \\
894,23(92,51 \%) \\
52,02(69,33 \%)\end{array}$ & $\begin{array}{r}2.012,66 \\
966,68 \\
75,03\end{array}$ \\
\hline
\end{tabular}


por exemplo, concluem que o tempo de recuperação do $\mathrm{V}_{2}$ é determinado mais pela intensidade do exercício executado do que por seu gasto energético total.

No que toca à diferença entre jovens e idosos, Chilibeck $e t$ $a l .{ }^{13}$ explicam que a recuperação mais rápida dos primeiros deve-se a maior capilarização muscular e a menor distância de difusão entre o oxigênio e os tecidos, quando isolados os efeitos do treinamento. Outros estudos reforçam tais considerações, indicando existir uma relação de natureza inversamente proporcional entre o tempo de recuperação do $\mathrm{VO}_{2}$ e a potência aeróbia dos grupos observados ${ }^{10,14,28}$.

\section{Recuperação da eliminação de gás carbônico}

As observações feitas para o oxigênio repetiram-se, em certa medida, para o padrão de eliminação do $\mathrm{CO}_{2}$. A exemplo do observado para o consumo de $\mathrm{O}_{2}$, a quantidade total de $\mathrm{CO}_{2}$ eliminado durante a recuperação aumenta com a intensidade do esforço (tabela 7). A participação relativa das duas fases de recuperação, contudo, parece ser mantida nas três IE. Os valores das constantes de velocidade $\alpha$ foram semelhantes nas três IE, em ambos os grupos. Assim, para o grupo jovem, te$\operatorname{mos} 1 / \alpha(100 \%)=67,8 \mathrm{~s}, 1 / \alpha(75 \%)=63 \mathrm{~s}$ e $1 / \alpha(40 \%)=$ 53,4 s. Para o grupo idoso, os valores de $1 / \alpha$ nas IE de $100 \%$, $75 \%$ e $40 \%$ foram, respectivamente, de $61,2 \mathrm{~s}, 59,4 \mathrm{~s}$ e $52,2 \mathrm{~s}$. Pode-se então aventar a hipótese de que, a exemplo do que acontece para o $\mathrm{V}_{2}$, seja qual for a intensidade do esforço e a idade, a constante de velocidade $1 / \alpha$ (relativa à fase rápida da curva de recuperação) para o $\mathrm{V} \mathrm{CO}_{2}$ será vizinha de 1 min.

A constante $1 / \beta$ no esforço máximo foi de $8,5 \mathrm{~min}$ para o grupo jovem e de $10,1 \mathrm{~min}$ para o grupo idoso. Para uma IE de $75 \%$ máx, o valor obtido para o grupo jovem foi de 5,9min e, para o grupo idoso, de $7,8 \mathrm{~min}$. Finalmente, as contantes para $40 \%$ IE máx foram de 10,4min (grupo jovem) e de $8,1 \mathrm{~min}$ (grupo idoso). Nota-se que os resultados para a constante lenta da recuperação do $\dot{\mathrm{V} C O}$, foram mais próximos, entre os grupos, do que o observado para o $\mathrm{VO}_{2}$. Apesar disso, em termos de recuperação total, o $\dot{\mathrm{V} C O}{ }_{2}$ pareceu ter sido mais dependente da intensidade do trabalho que o $\dot{\mathrm{V}}_{2}$. A tabela 7 mostra que os valores de $\dot{\mathrm{V}} \mathrm{CO}_{2}$, nos dois grupos etários, foram mais dispersos quando consideradas as três IE.

Nota-se ainda que a quantidade total de $\mathrm{CO}_{2}$ eliminado foi semelhante para os jovens e idosos, em cada IE. Como as constantes $1 / \beta$, ao menos em nosso modelo matemático, tenderam a ser maiores para os idosos nas intensidades superiores, poder-se-ia imaginar que, apesar de recuperação total equivalente, este grupo teria eliminação menos eficaz, exigindo mais tempo para levar a $\mathrm{pCO}_{2}$ sanguínea aos patamares de repouso. Nossas observações concordam com as de Takahashi et al. ${ }^{29}$. Além de indicarem dependência maior da fase lenta de recuperação à intensidade do esforço, esses autores observaram que o declínio do $\dot{\mathrm{V}} \mathrm{CO}_{2}$ era estreitamente proporcional ao declínio da ventilação pulmonar, mas que a cinética da primeira variável era maior.
Em outras palavras, o tempo de recuperação do gás carbônico pode ser menor do que o da $\mathrm{V}_{\mathrm{E}}$, como demonstrado nas tabelas 5 e 6 . Isso significa que a influência do $\mathrm{CO}_{2}$ sobre os quimiorreceptores centrais não é o único fator que pressiona a ventilação na fase de recuperação, que pode ser estimulada por outras vias, como comando central ou neurônios aferentes provenientes da musculatura utilizada. Não estamos, porém, em condições de confirmar essas hipóteses.

\section{Recuperação da ventilação pulmonar}

Os valores da constante $\alpha$ para o grupo jovem exibiram comportamento parecido àquele observado para o $\dot{\mathrm{VO}}_{2}$ e o $\dot{\mathrm{V} C \mathrm{CO}_{2}}$, isto é, nas diferentes intensidades de esforço existiria a possibilidade de uma constante única próxima de $1 \mathrm{~min}$. Já para o grupo idoso, a relação $1 / \alpha$ se afastou desse padrão: os valores obtidos nas diferentes intensidades de esforço foram de 111,6s (IE 100\%), 94,8s (IE 75\%) e 76,8s (IE 40\%). No entanto, a recuperação total para a constante rápida (tabela 7), em ambos os grupos, foi semelhante. Para a constante de recuperação lenta a situação é muito menos precisa; por exemplo, seu valor a IE $40 \%$ para o grupo jovem foi de $0,020 \pm 0,022 \mathrm{e}$, para IE $100 \%$ no grupo idoso, de $0,055 \pm 0,020$. Tais desviospadrões limitam as possibilidades de inferências. De qualquer forma, parece ser mais fácil detectar influências da intensidade do esforço sobre as constantes $\beta$ para o grupo idoso que para o grupo jovem (tabelas 5 e 6 ). Tanto quanto para o componente $\mathrm{A} / \alpha$, a recuperação total do componente lento $(B / \beta)$ foi equivalente para jovens e idosos. A quantidade total de ar ventilado durante a recuperação aumenta com a IE (tabela 7). Nas três intensidades observadas, o componente lento tem influência preponderante sobre o excedente de ventilação, intervindo na mesma proporção para ambos os grupos (entre 70 e $80 \%$ ).

Alguns comentários podem ser feitos com base nesses resultados. Em primeiro lugar, há certa coerência entre o exposto aqui e os resultados obtidos para o $\mathrm{VCO}_{2}$. Se os idosos são menos eficazes para a eliminação do gás carbônico, parece lógico que sua $V_{E}$ permaneça elevada mais tempo que nos jovens (o que é atestado pelos valores da contante $\beta$ ), mesmo que seu $\mathrm{VCO}_{2}$ total seja semelhante. Poderíamos então conjecturar que as diferenças entre os indivíduos jovens e idosos, no que diz respeito à ventilação pulmonar, poderiam dever-se a três fatores principais: a) menor potência aeróbia máxima nos idosos, acarretando maior acúmulo de lactato em atividades intensas e menor capacidade de metabolização durante a recuperação; b) menor eficácia do sistema tampão respiratório; c) menor eficácia dos mecanismos de termorregulação. Algumas dessas possibilidades foram consideradas por Chick et al. ${ }^{24}$, comparando a recuperação do $\mathrm{VO}_{2}, \dot{\mathrm{VCO}}_{2}, \mathrm{~V}_{\mathrm{E}}$ e FC em indivíduos jovens e idosos, após exercitação a IE 70\%. Os autores observaram que o período de recuperação foi sistematicamente maior para o grupo idoso, propondo que isso 
seria consistente com as menores potência aeróbia máxima e sensibilidade dos quimiorreceptores ao $\mathrm{CO}_{2}$ desses indivíduos.

\section{Recuperação da freqüência cardíaca}

Contrariamente ao exposto até aqui, os valores das constantes $\alpha$ e $\beta$ associados à recuperação da FC dependem indiscutivelmente da intensidade do esforço realizado. Maior a IE, mais os valores $1 / \alpha$ e $1 / \beta$ alongam-se (tabelas 5 e 6 ). Assim, para o grupo jovem temos $1 / \alpha=120,6$ s (IE $100 \%$ ), $1 / \alpha=69$ s (IE $75 \%$ ) e $1 / \alpha=40,2$ s (IE $40 \%$ ). Os resultados para a constante lenta no grupo jovem foram $1 / \beta=71,4 \mathrm{~min}$ (IE $100 \%$ ), $1 / \beta=27,0 \mathrm{~min}(\mathrm{IE} 75 \%$ ) e $1 / \beta=4,8 \mathrm{~min}$ (IE $40 \%$ ). Para os idosos, os valores observados para a constante rápida foram $1 / \alpha=166,2 \mathrm{~s}(\operatorname{IE} 100 \%), 1 / \alpha=146,4 \mathrm{~s}(\mathrm{IE} 75 \%)$ e $1 / \alpha=105 \mathrm{~s}$ (IE $40 \%$ ). Para a constante lenta temos $1 / \beta=76,9 \mathrm{~min}$ (IE $100 \%$ ) e $1 / \beta=66,7 \mathrm{~min}$ (IE $75 \%$ ). Os resultados para IE $40 \%$ foram por demais imprecisos, o que restringe a possibilidade de discuti-los $(\beta=0,022 \pm 0,053)$.

A FC é o parâmetro que, nos primeiros minutos de recuperação, demonstra as maiores diferenças em função da IE observada. Igualmente, trata-se da variável cuja recuperação é mais lenta, retornando aos valores de repouso depois de todas as outras (tabelas 5 e 6). Notamos que, para o grupo idoso, os componentes lentos de recuperação nas intensidades de $100 \%$ e $75 \%$ do máximo foram muito mais semelhantes que no grupo jovem. Isso pode dever-se ao fato de que os idosos têm menor potencial de elevação da $\mathrm{FC}^{1,24,27,30}$.

Assim, em atividades submáximas de intensidade moderada a alta, esse grupo já exibiria valores de FC próximos à freqüência máxima, o que é menos provável em indivíduos jovens. Notamos ainda que, em termos de recuperação total (tabela 7), nas intensidades superiores o componente lento é praticamente responsável por quase toda a normalização da FC (cerca de $90 \%$ da recuperação). Já em atividades de baixa intensidade (IE 40\%), a participação do componente rápido aumenta em importância, aproximando-se do patamar dos $30 \%$. Tal comportamento parece não depender da idade dos indivíduos.

Digno de atenção especial é o fato de que, das quatro variáveis observadas, a FC foi aquela cujo período de recuperação foi mais longo. Os estudos disponíveis são conflitantes a esse respeito. Por exemplo, Williams e Horvath ${ }^{7}$ propõem que, após 30 a 40 minutos de exercício de intensidade moderada, $\dot{\mathrm{VO}}_{2}$, $\dot{\mathrm{VCO}}_{2}$ e $\mathrm{V}_{\mathrm{E}}$ tenham seus valores normalizados após cerca de 10 minutos, enquanto a FC permaneceria elevada por $30 \mathrm{mi}-$ nutos. Já Chick et al. ${ }^{24}$, analisando a recuperação após exercício a IE 70\%, sugerem que, após 10 minutos de repouso, o $\dot{\mathrm{VO}_{2}}, \dot{\mathrm{VCO}_{2}}$ e FC se normalizem, mas que a $\mathrm{V}_{\mathrm{E}}$ permaneça alta durante mais tempo. Nossos resultados aproximam-se mais dos apresentados por Williams e Horvath ${ }^{7}$, apesar dos tempos de recuperação estimados pelas curvas de exponenciais calculadas terem sido maiores do que os relatados por esses autores.
Conclui-se que a recuperação da $\mathrm{FC}$ não é inteiramente determinada pela recuperação do $\dot{\mathrm{VO}}_{2}$. $\mathrm{O}$ padrão de comportamento da $\mathrm{FC}$ não acompanha o do $\mathrm{VO}_{2}$, principalmente na fase rápida de recuperação. Na fase lenta, apesar de a influência da IE fazer-se sentir de forma semelhante ao observado para o $\dot{\mathrm{VO}}_{2}$, as constantes $1 / \beta$ implicam tempo de recuperação muito mais longo para a FC. Outros fatores, como eliminação do $\mathrm{CO}_{2}$, concentração de catecolaminas, regulação térmica ou reposição de substratos utilizados (por exemplo, creatina-fosfato) devem influenciar seus valores. Esse efeito é ainda maior para a pessoa idosa - em IE $75 \%$ o tempo de recuperação $1 / \beta$ em GI foi de $67 \mathrm{~min}$, contra $27 \mathrm{~min}$ em GJ. De acordo com os resultados discutidos até aqui, isso poderia ser explicado tanto por uma potência aeróbia máxima mais reduzida, quanto pelo fato de que os idosos levam mais tempo para eliminar o $\mathrm{CO}_{2}$ produzido na atividade.

\section{CONCLUSÕES}

1) Considerando modelos com uma ou duas exponenciais, as curvas de recuperação para as quatro variáveis observadas exibiram o melhor ajustamento para uma soma de duas exponenciais;

2) Quanto maior a intensidade do esforço, maior o percentual de contribuição da fase lenta de recuperação para a normalização das variáveis observadas, fato que independe da idade dos sujeitos. O envelhecimento parece não afetar a participação relativa das fases da recuperação;

3) Pode existir uma constante comum para a chamada 'fase alática' da curva de recuperação do oxigênio e do gás carbônico, independentemente da intensidade do esforço e mesmo da faixa etária;

4) A influência da intensidade do esforço na fase lenta de recuperação do oxigênio e do gás carbônico foi mais sentida pelo grupo idoso. Isso pode advir da diminuição da potência aeróbia máxima com a idade, assim como de menor sensibilidade à concentração do $\mathrm{CO}_{2}$;

5) O padrão da fase rápida da recuperação da ventilação pulmonar foi semelhante ao observado para o $\dot{\mathrm{VO}}_{2}$ e $\dot{\mathrm{V}} \mathrm{CO}_{2}$ no grupo jovem. No grupo idoso, ela afastou-se desse padrão, sendo mais lenta. A intensidade do esforço parece afetar mais o componente lento da curva de ventilação no idosos que nos jovens, ainda que a recuperação total tenha sido equivalente. Isso sugere que o sistema tampão ventilatório do idoso é menos eficaz que nos indivíduos mais jovens;

6) As fases rápida e lenta da recuperação da frequiência cardíaca dependem da intensidade do esforço, sendo mais longas se o esforço é maior. Trata-se da variável cuja curva de recuperação foi mais lenta, o que foi ainda mais sentido no grupo idoso. Atribui-se esse particular ao fato de que esse grupo possui menor potencial de elevação da freqüência cardíaca. Nas intensidades de $100 \%$ e $75 \%$ da carga máxima de trabalho, a fase lenta da recuperação é responsável por quase toda 
a normalização da freqüência cardíaca. A 40\% da carga máxima, a participação do componente rápido aumenta, aproximando-se do patamar dos $30 \%$. Esse padrão parece não ser afetado pela idade.

\section{AGRADECIMENTOS}

Agradecemos ao Dr. Cláudio Gil Soares Araújo pelos comentários e sugestões. Apoio financeiro parcial do CNPq, processo 200063/ 94-4.

\section{REFERÊNCIAS}

1. Åstrand I. Aerobic work capacity in men and women with special reference to age. Acta Physiol Scand 1960;49(Suppl 169):1-92.

2. Dehn MM, Bruce RA. Longitudinal variations in maximal oxygen intake with age and activity. J Appl Physiol 1972;33:805-7.

3. Hagberg JM. Effect of training on the decline of $\mathrm{VO}_{2}$ with aging. Fed Proc 1987;46:1830-3.

4. Kohrt WN, Malley MT, Coggan AR, Spina RJ, Ogawa T, Ehsani AA, et al. Effects of gender, age and fitness level on response of $\mathrm{VO}_{2}$ max training in 60-70 yr olds. J Appl Physiol 1991;71:2004-11.

5. Kasch FW, Boyer JL, Van Camp SP, Verity LS, Wallace JP. Effects of exercise on cardiovascular ageing. Age Ageing 1993;22:5-10.

6. Pollock ML, Mengelkoch LJ, Graves JE, Lowenthal DT, Limacher MC, Foster C, Wilmore JH. Twenty-year follow-up of aerobic power and body composition of older track athletes. J Appl Physiol 1997; 82: 1508-16.

7. Williams RE, Horvath SM. Recovery from dynamic exercise. Am J Physiol 1995;268(6 Pt 2):H2311-20.

8. McLellan TM, Skinner JS. Blood lactate removal during active recovery related to the aerobic threshold. Int J Sports Med 1982;3:224-9.

9. Bogdanis GC, Nevill ME, Lakomy HK, Graham CM, Lovis G. Effects of active recovery on power output during repeated maximal sprint cycling. Eur J Appl Physiol 1996;74:461-9.

10. Nummela A, Rusko H. Time course of anaerobic and aerobic energy expenditure during short-term exhaustive running in athletes. Int J Sports Med 1995;16:522-7.

11. Dawson B, Straton S, Randall N. Oxygen consumption during recovery from prolonged submaximal cycling below the anaerobic threshold. J Sports Med Phys Fitness 1996;36:77-84.

12. Short KR, Wiest JM, Sedlock DA. The effect of upper body exercise intensity and duration on post-exercise oxygen consumption. Int $\mathrm{J}$ Sports Med 1996;17:559-63.

13. Chilibeck PD, Paterson DH, Cunningham DA, Taylor AW, Noble EG. Muscle capillarization, $\mathrm{O}_{2}$ diffusion distance and $\dot{\mathrm{VO}}_{2}$ kinetics in old and young individuals. J Appl Physiol 1997;82:63-9.
14. Short KR, Sedlock DA. Excess post-exercise consumption and recovery rate in trained and untrained subjects. J Appl Physiol 1997;873: 153-9.

15. Hill AV, Long CN, Lipton H. Muscular exercise, lactic acid and the supply and utilisation of oxygen. Parts I-III. Proc R Soc 1924;B96: 438-75.

16. Hill AV, Long CN, Lipton H. Muscular exercise, lactic acid and the supply and utilisation of oxygen. Parts IV-VI. Proc R Soc 1925;B97: 84-137.

17. Margaria R, Edwards HT, Dill DB. The possible mechanism of contracting and paying the oxygen debt and the role of lactic acid in muscular contraction. Am J Physiol 1933;106:689-715.

18. Henry FM. Aerobic oxygen consumption and alactic debt in muscular work. J Appl Physiol 1951;3:427-38.

19. Henry FM, De Moor JC. Lactic and alactic oxygen consumption in moderate exercise of graded intensity. J Appl Physiol 1956;8:608-14.

20. Margaria R, Ceretelli P, Di Prampero PE, Massari C, Torelli G. Kinetics and mechanism of oxygen debt contraction in man. J Appl Physiol 1963;18:371-7.

21. Katch VL, Girandola RN, Henry FM. The influence of the estimated oxygen cast of ventilation on oxygen deficity and recovery oxygen intake for moderately heavy bicycle ergometer exercise. Med Sci Sports Exerc 1972;4:71-6.

22. Katch VL. Kinetics of oxygen uptake and recovery for supramaximal work of short duration. Int Z Angew Physiol 1976;31:197-207.

23. Joye H. Comparaison des composantes rapides et lentes de la courbe de consommation d'oxygène, d'élimination d'anhydride carbonique et de la courbe de ventilation pendant la période de récupération. Int $\mathrm{Z}$ Angew Physiol 1968;25:210-23.

24. Chick TW, Cagle TG, Vegas FA, Poliner JK, Murata GH. The effect of aging on submaximal exercise performance and recovery. J Gerontol 1991;46:B34-8

25. American College of Sports Medicine. Guidelines for exercise testing and prescription. $5^{\text {th }}$ ed. Philadelphia: Lea \& Febiger, 1995.

26. Åstrand PO, Bergh U, Kilbom A. A 33-year follow-up of peak oxygen uptake and related variables of former physical education students. J Appl Physiol 1997;82:1844-52.

27. Zwart BCH, Frings-Dresen MHW, Van Dijk FJH. Physical workload and the ageing worker: a review of the literature. Int Arch Occup Environ Health 1995;68:1-12.

28. Menghini F, Dally L, Fazzini PF, Menotti A, Prati PL, Rovelli F, Antoniucci D, Seccareccia F. Normal behaviour of circulatory parameters during exercise - Reference values for heart rate and systemic blood pressure. G Ital Cardiol 1995;25:967-75.

29. Takahashi T, Niizeki K, Miyamoto Y. Respiratory responses to passive and active recovery from exercise. Jpn J Physiol 1997;47:59-65.

30. Docherty JR. Cardiovascular responses in ageing: a review. Pharmacol Rev 1990;42:103-25. 